\title{
The NCCN Clinical Practice Guidelines in Oncology: A Primer for Users
}

\author{
Rodger J. Winn, MD, and Joan McClure, MS, Rockledge, Pennsylvania
}

\begin{abstract}
Key Words
Practice guidelines, medical oncology, outcome and process assessment, neoplasms, health planning, NCCN
\end{abstract}

\begin{abstract}
Guidelines are becoming increasingly important as potential tools in improving clinical decision making. Because oncology practice encompasses a large number of tumors and their variants and because each tumor is characterized by heterogeneous presentations and clinical evolutions, an oncology guidelines program must be large in scope. Oncology practice is slowly moving toward evidencebased status, but guideline developers still must rely on less than perfect information to achieve this scope. By formalizing the consensus process, the NCCN program relies on the expertise of a broad range of cancer specialists to interpret the major clinical studies and apply their evaluative skills in assessing the relevance of these studies to clinical practice. In areas in which data are meager or contradictory, these experts are still charged with making recommendations if they believe their collective clinical experience points to a reasonable approach to disease management. It follows, therefore, that guidelines represent one of the most dynamic areas in medicine. The annual review process is designed to incorporate change as new evidence or innovative therapies become available. Therefore, the guidelines should be a true reflection of the state-of-the-art in oncology. The ultimate goal, as always, is improving care for the cancer patient (JNCCN 2003;1:5-13).
\end{abstract}

Over the past decade, the health delivery system has become increasingly concerned with improving the level of care provided to its patient populations and ensuring that all patients receive preventive, diagnostic, treatment, and supportive services that are most likely to lead to optimal outcomes. ${ }^{1}$ These concerns have become an area of focus within the oncology community. ${ }^{2}$ Clinical practice

From the National Comprehensive Cancer Network, Rockledge, Pennsylvania.

Received on September 20, 2002; accepted for publication October 1, 2002.

Correspondence: Rodger J. Winn, MD, JNCCN, 50 Huntingdon Pike, Suite 200, Rockledge, PA 19046. E-mail: winn@nccn.org guidelines have become increasingly available, and the oncology community has begun to use and assess guidelines as possible aids in improving care, ${ }^{3,4}$ however, as with all new tools, the user must become familiar with their design, capabilities, and limitations to ensure that they produce the best results and, most importantly, that they cause no harm. ${ }^{5}$

The NCCN Clinical Practice Guidelines in Oncology are a comprehensive set of guidelines detailing the sequential management decisions and interventions for the malignancies that affect $95 \%$ of all cancer patients (Table $1)$. In addition, separate guidelines discuss major prevention and screening subjects and the major supportive care areas. Each guideline consists of an algorithm or decision pathway outlining care management, a manuscript discussing important issues related to the algorithm, and references providing data on which recommendations are based. These guidelines are developed and updated by 45 individual panels, composed of over 600 clinicians and oncology researchers from the $19 \mathrm{NCCN}$ member institutions and their affiliates.

This introductory article provides an overview of some of the major issues that guideline developers and users must address and discusses the purpose and development of the guidelines and some guides to interpretation. Our goal is to increase the likelihood that these pathways will effectively assist clinicians in patient treatment and ultimately improve the level of care in the oncology community. ${ }^{6}$

\section{Definitions}

The most widely quoted definition of a clinical practice guideline is "a systematically developed statement to assist practitioner and patient decisions about appropriate health care for specific clinical circumstances." In the taxonomy of clinical decision-making aids, 3 levels have 
Winn and McClure

Table 1 NCCN Clinical Practice Guidelines in Oncology

Treatment of Cancer by Site

Acute Myeloid Leukemia

Bladder Cancer

Bone Cancer

Breast Cancer

Central Nervous System Tumors

Cervical Cancer

Chronic Myelogenous Leukemia

Colon Cancer

Rectal Cancer

Anal Canal Cancer

Esophageal Cancer

Gastric Adenocarcinoma

Head and Neck Cancers

Hepatobiliary Cancers

Hodgkin's Disease

Kidney Cancer

Melanoma

Myelodysplastic Syndromes

Multiple Myeloma

Neuroendocrine Tumors

Non-Hodgkin's Lymphoma

Non-Melanoma Skin Cancer

Non-Small Cell Lung Cancer

Occult Primary Cancer

Ovarian Cancer

Pancreatic Cancer

Prostate Cancer

Sarcoma

Small Cell Lung Cancer

Testicular Cancer

Thyroid Cancer

Uterine Cancers

Detection, Prevention, and Risk of Cancer

Breast Cancer Risk Reduction

Breast Cancer Screening and Diagnosis

Cervical Cancer Screening

Colorectal Cancer Screening

Genetic/Familial High-Risk Screening

Prostate Cancer Early Detection

Supportive Care

Antiemesis

Cancer and Treatment-Related Anemia

Cancer-Related Fatigue

Cancer Pain

Distress Management

Fever and Neutropenia

Palliative Care been recognized: standards, guidelines, and options. ${ }^{8}$ Standards exist when there is incontrovertible evidence that a specific action is indicated. In almost all situations, not performing the standard intervention constitutes a breach of good medical care. Guidelines are a less rigid set of parameters and exist when available data point to interventions that are probably applicable to most, but not all, patients. Guidelines are recommendations; therefore, the caveat always exists that clinical judgment and patient preference must be considered to determine whether the recommendation applies to a particular management decision. ${ }^{9}$ The third level, options, applies when no data exist to support a clinical decision. On this level, possible choices are provided, any of which may used based on clinical judgment.

The NCCN Clinical Practice Guidelines in Oncology fall into the guideline category. The scope of each guideline is intended to be comprehensive. The aim is to incorporate into the management schema many of the major clinical scenarios that are frequently encountered across the entire spectrum of the disease. ${ }^{10}$ Obviously, given the multiple factors that define these scenarios, only the major elements can be accounted for in developing the decision pathways. Special circumstances may always apply that render the recommended intervention invalid. In the simplest scenario, surgical excision of a stage II lung cancer may not be appropriate for a patient with severely disabling chronic obstructive pulmonary disease. In this instance, the concept of appropriateness, the evaluation that the chances of benefit sufficiently outweigh the risks to make the intervention worthwhile, ${ }^{11}$ becomes exceptionally important.

At the more oncology-specific level, it is also true that the guidelines cannot provide firm recommendations for all of the possible clinical situations that require medical decision making. As every practicing oncologist knows, rare, or at least uncommon, clinical situations are encountered frequently. Trying to incorporate recommendations for the entire range of rare scenarios into the pathways would complicate them beyond usability. Unfortunately, for many of these rare situations, the data needed for making firm recommendations are usually not available, and individual approaches may vary. ${ }^{12}$ For this reason, the NCCN panels have adopted the " $\% \%$ rule," which dictates that clinical scenarios representing less than $5 \%$ of cases for a specific tumor will typically not be cov- 
ered in the algorithm. Important exceptions can occur if a there are major implications for managing a rare situation and sufficient data exist to make a sound recommendation. For example, a pathway for hepatic resection of colorectal metastases is included, even though this comprises only $3 \%$ of cases; however, this criterion may be too inclusive because a level of $20 \%$ to $25 \%$ of affected patients has been proposed as the threshold for creating a pathway. ${ }^{13}$

Given the heterogeneity of factors that may be used to define a particular clinical scenario, a guideline path may not branch to encompass all the clinical permutations. Instead, the guideline will list options for the practitioner to use based on his or her evaluation of the individual circumstances. ${ }^{14}$ Thus, the head and neck cancer guideline recommends either total laryngectomy or hemilaryngectomy for the management of a pyriform sinus lesion. The decision of which procedure to employ is based on, among other things, the size of the tumor, whether it is medial or lateral, fixed or not fixed, and exophytic or invasive. Including all permutations of these factors would require at least 16 separate pathways, and even then, the situation would still probably require individualized decision making based on the nuances of the clinical findings.

For all of these reasons, therefore, the NCCN guideline program should be viewed as recommendations intended to apply to a large number of cancer patient circumstances. They must always be interpreted in the light of the individual situation.

\section{Guideline Purposes}

As basic tools, clinical practice guidelines can aid change in the health care system at 3 levels: the individual practitioner or provider level, the institutional or group level, and the societal or governmental level. ${ }^{15}$ The basic premise at each of these levels is that health care will be improved if variation in care that does not reflect clinically based imperatives is minimized. ${ }^{16}$ That this variation in care exists has been well documented in oncology. ${ }^{2}$ Examples of variation in care may occur at all phases of tumor management ranging from diagnostic or follow-up testing ${ }^{17}$ to primary and adjuvant treatment ${ }^{18,19}$ to salvage $e^{20}$ and palliative therapies. $^{21}$

On an individual level, guidelines can serve as educational roadmaps to assist in decision making for the individual patient. ${ }^{22}$ The explosion of medical information over the past several years has been enormous, and it is virtually impossible for the busy clinician to remain current on the voluminous literature. ${ }^{23}$ Guidelines, when updated on a regular basis, are a method whereby the oncologist can keep apprised of new developments and approaches to disease management. ${ }^{24}$

In keeping with the fundamental guideline definition of assisting patient and physician decision making, several programs have developed patient counterparts to the physician guidelines..$^{25,26}$ The NCCN Guidelines for Patients program, in collaboration with the American Cancer Society, has developed patient guidelines for the major tumors and symptom-management pathways (Table 2 ). These patient guidelines use the same pathways as the physician guidelines, but include language that is more understandable to the lay public. The guidelines for patients provide a glossary and the necessary explanatory text to walk the patient through the disease management plan. Essentially, allowing the patient and the physician to be "on the same page" can be a powerful tool to facilitate shared decision making. ${ }^{27}$

On the institutional level, a major concern is that variation represents a compromise in the quality of care and that rectifying this through programs such

Table 2 NCCN/ACS Treatment Guidelines for
Patients
Cancer Treatment Guidelines
Breast Cancer
Cáncer de Seno (Breast Cancer)
Colon and Rectal Cancer
Cáncer de Colon y Recto (Colon and Rectal Cancer)
Lung Cancer
Melanoma
Ovarian Cancer
Prostate Cancer
Cáncer de la Próstata (Prostate Cancer)
Supportive Care Treatment Guidelines for Patients
Cancer Pain
El Dolor Asociado con el Cáncer (Cancer Pain)
Cancer-Related Fatigue
Fever and Neutropenia
Nausea and Vomiting
Náuseas y Vómitos (Nausea and Vomiting)


as Total Quality Management Improvement can benefit patients. ${ }^{28}$ One method of evaluating the quality of care is by assessing the process: what was done to the patient. ${ }^{29}$ If these processes have been proven to lead to better patient outcomes (validated), we can assume that the degree to which they are followed predicts for better outcomes. Clinical practice guidelines are process maps, and insofar as the recommendations are supported by sound evidence of clinical benefit, adhering to them may be viewed as a quality measure. ${ }^{30}$ Remeasuring adherence after remedial interventions can then be used as quality improvement. The NCCN Outcomes Database program ${ }^{31}$ is now tracking concordance to the breast cancer treatment algorithm in over 10,000 cases of the disease in 13 member institutions and has initiated outcomes databases to track concordance to the non-Hodgkins lymphoma and pain guidelines as well. The outcomes database also provides reports to participating NCCN institutions to allow benchmarking against aggregate patterns of care and outcomes in NCCN institutions.

On a larger societal level, including governmental and third-party payer concerns, the issues include policy or resource allocation. In this sphere, guidelines can be used to define acceptable practice ${ }^{32}$ and establish high-level benchmarks for determining the care level across broad groups. ${ }^{33}$ One proposed use of guidelines at this level is for diminishing the cost of care. ${ }^{34}$ Unfortunately, the capacity of guideline implementation to accomplish this is still largely unproven. ${ }^{35}$ If unwarranted variation results from overuse or inappropriate use of expensive procedures, instituting guidelines may curtail excessive costs. ${ }^{36} \mathrm{We}$ must also recognize that if the broad institution of guideline use corrects serious underuse, up-front costs may rise, although the reduction of subsequent disease mortality or morbidity may lead to overall savings. ${ }^{37}$

\section{Guideline Development}

Researchers have described four methods of guideline development: informal consensus, formal consensus, evidence based, and explicit. ${ }^{38}$ In informal consensus, no explicit rules govern what evidence will be reviewed or what process or criteria will be used to evaluate it. In formal consensus, such as the National Institutes of Health Consensus panels, the group may all use the same data, but the evaluation process is not specified. In another variant of this process, formal methods of repetitive review may be used. ${ }^{39}$ The explicit approach uses quantification of clinical benefit and costs and has been used in oncology primarily in developing screening guidelines. ${ }^{40}$

Most guidelines experts endorse evidence-based guideline development ${ }^{41}$ and instruments to measure guideline quality incorporate several ratings of the evidence-based process. ${ }^{42}$ This rigorous method is based on review of all data, with the strength of the evidence rated based on the research design that yielded the data. Meta-analyses and high-powered randomized clinical trials are the highest level..$^{43}$ Systematic reviews that rank the evidence and collate the data can be extremely useful in assisting the guideline process. ${ }^{44}$

The evidence-based process is not simply "cranking out" recommendations based on independent analytic function. Rather, the knowledge and experience of expert clinicians form the basis for interpreting and evaluating which data is pertinent. ${ }^{45}$ The analytic process is often imperfect at best. For many of the major clinical decisions facing the oncologist, high-level data do not exist. Djulbegovic and Hadley ${ }^{46}$ examined the level of evidence for 154 recommendations made in hematologic malignancy guidelines and found that only $24 \%$ of recommendations were backed up by randomized clinical trials. The remaining recommended interventions were based on lower levels of evidence (21\%) or clinical experience (55\%). The evidencebased process is further complicated by contradictory evidence or studies of questionable generalizability.

Given the scope of the NCCN guidelines-the entire spectrum of clinical decisions for virtually all tumor sites- the NCCN panels use a consensus process based on expert knowledge and analysis of the most pertinent data. ${ }^{47}$ Many of the panels incorporate formal presentations of data for specific controversial issues into the guideline meetings. Because the panels are composed of tumor specialists from each of the member institutions, panel members have in-depth knowledge of the literature and awareness of, if not actual participation in, the trials that provide the evidence for the formulations. Nevertheless, formal systematic reviews are not undertaken, and thus, the standards for an evidence-based process cannot be met. Analysis of the consensus processes has shown that different panels can in fact generate similar sets of recommendations. ${ }^{48}$ For example, a comparison of the management recommendations for advanced lung cancer $^{49}$ showed that they did not appear to be signif- 
icantly different from the recommendations in the more evidence-based ASCO guidelines ${ }^{50}$ and the NCCN lung cancer guidelines. ${ }^{51}$

The major concern about a consensus-based process is potential bias; ${ }^{52}$ however, formal processes using stepwise review and consensus-building exercises are indispensable in minimizing this difficulty. ${ }^{53}$ Without these explicit methods for dealing with panel members with disparate views and interests, the group may not accomplish its mission. ${ }^{54}$ The other major step in eliminating bias is to ensure that all relevant stakeholders in the guideline are represented. Singlediscipline panels will create different sets of guideline recommendations than multidisciplinary panels. ${ }^{55}$ Single-specialty societies may generate unifocal guidelines that reflect the biases of the developers and that cannot gain the acceptance of the broad medical community. ${ }^{56}$

\section{NCCN Guidelines Development and Review}

Each NCCN member institution nominates a representative to the NCCN Guidelines Steering Committee. These steering committee members nominate panel members from their faculties for each guideline panel. With the scope of the cancer treatment programs at NCCN member institutions, panel members are specialists for the particular tumor under consideration. Discussion with the panel chair and the NCCN guidelines staff ensures that all pertinent disciplines are represented, and each panel has its own distinct membership based on the requirements for comprehensive input for that topic. Therefore, panels such as central nervous system, breast, prostate screening, and lymphoma have active pathologist participation. In some areas, such as myelodysplastic syndromes and distress, panels include experts from nonmember institutions members. Equally important, the panel chair ensures that if there are major conflicts in how the cancer is approached, proponents of each approach are included. For example, special care was taken to ensure that the Thyroid Cancer Panel included both proponents of lobectomy and those of total thyroidectomy. Another important aspect of panel composition is the inclusion of lay members on panels in which their input is essential. This assures that the consideration of patient preferences plays a role in evolving pathway recommendations.
The NCCN development and review process is built around a system of iteration and feedback. ${ }^{57}$ For new guidelines, the panel develops a preliminary draft that is then circulated through all of the member institutions for comments. These comments are collated by NCCN staff and serve as the basis for a second panel meeting to modify the pathways based on broad input from the NCCN faculties. This external validation process incorporates the ideas of a larger pool of physicians and others into the process and ensures that a broad representation of views is used and that inadvertent biases are recognized and corrected.

An important aspect of any effective guideline process is review and updating. ${ }^{58}$ An analysis of the Agency for Healthcare Research and Quality guidelines revealed that 7 of 17 required major updating. ${ }^{59}$ The study recommended that guidelines be updated at least every 3 years to remain valid. The NCCN program, however, has a more rigorous program of annual review. The Guidelines Steering Committee believes that the dynamic research programs in oncology necessitate annual guideline re-examination to ensure that algorithms reflect state-of-the-art care. The annual update process also uses iteration and review, beginning with the panel members distributing the current year's pathway to institutional colleagues. The NCCN staff collates their critiques and comments, and these form the basis for the annual panel meeting. Each guideline undergoes a full-day, on-site panel meeting review at least once every 3 years and review by teleconference in the interim years (some panels meet onsite every year). At these meetings, panel members discuss and evaluate new data to ensure all new findings are incorporated into the guideline. In addition, the panel considers each institutional recommendation and decides whether to modify the guideline based on it. The panel also records the level of consensus and provides appropriate supporting references to guidelines staff to include in the bibliography.

After the panel meeting, guidelines staff update the algorithm to reflect the panel's deliberations and send it to the panel chair for approval. If major changes have been made, the revised algorithm is again circulated to the entire panel. A writing committee of panel members updates the accompanying manuscripts, which highlight major issues in disease management, describe treatment alternatives, and discuss data that support the recommendations. 
The concurrent online publication of the guidelines (www.nccn.org) allows the NCCN to update guidelines more frequently if important new data are published between normal annual update cycles. In this situation, the panel chair requests a meeting or teleconference to discuss the new data; changes are proposed, and if the panel adopts the changes, the guideline is updated. An interim update can be accomplished in as little as 2 to 3 weeks following release of new data. The most recently updated version of each guideline is posted on NCCN's website.

\section{Conflict of Interest}

The relationships between panel members and the pharmaceutical industry can be viewed as a potential source of bias in guideline development. ${ }^{60}$ As faculty at large academic cancer centers, most panel members are involved in clinical research, and many have active relationships with sponsors developing new therapies. Therefore, an explicit procedure for dealing with potential conflicts was deemed necessary. ${ }^{61}$ At the beginning of each panel meeting, individual members disclose potential conflicts of interest, including research support, service on an advisory committee, speakers' bureau participation, and equity holdings. The panel may exclude a member from the discussion of areas in which they have a major conflict of interest. Aggregate panel disclosures are published with each guideline.

Although pharmaceutical companies and other interested parties can submit scientific data and comments for panel consideration, they have no control over the panel's recommendations. Industry representatives do not participate in panel deliberations.

As an organization, NCCN accepts general sponsorship grants from multiple pharmaceutical and biotechnology companies and grants for the support of specific projects; however, no pharmaceutical industry support is accepted for any component of guideline development. Pharmaceutical industry support is accepted in the form of educational grants for presentation of guidelines updates at NCCN meetings and for dissemination of the guidelines.

\section{Categories of Consensus}

Guidelines consist of management recommendations. Each recommendation is based on clinical trial results, expert evaluation and interpretation, outcomes analyses, and clinical experience, which are, in essence, the rationale for each decision. This reasoning should be transparent so that the guideline users understand how and why a panel made recommendations. To an extent, the manuscript serves this function.

As an additional aid, guidelines use a rating system to inform the user of the strength of each recommendation. ${ }^{62}$ Most evidence-based systems use a scale to rate the strength of the evidence and, based on this level, the strength of the recommendation, although applying these scales can be difficult. ${ }^{63}$ Because the NCCN guidelines are consensus based, the Categories of Consensus rating system is used. These categories, which are listed with each guideline, include two dimensions: the strength of the evidence and the degree of consensus. The interpretation of these categories is as follows:

- Category 1: The recommendation is based on highlevel evidence (ie, high-powered randomized clinical trials or meta-analyses), and the panel has reached uniform consensus that the recommendation is indicated. In this context, uniform means near unanimous positive support with some possible neutral positions.

- Category 2A: The recommendation is based on lower level evidence, but despite the absence of higher level studies, there is uniform consensus that the recommendation is appropriate. Lower level evidence is interpreted broadly and runs the gamut from phase II or large cohort studies ${ }^{64}$ to individual practitioner experience. Importantly, in many instances, the retrospective studies are derived from clinical experience of treating large numbers of patients at a member institution, and thus, panel members have first-hand knowledge of the data. Inevitably, some recommendations must address clinical situations for which limited or no data exist. In these instances, the congruence of experience-based opinions provides an informed if not confirmed direction for optimizing patient care. These recommendations carry the implicit recognition that they may be superseded as higher level evidence becomes available or as outcomes-based information becomes more prevalent. ${ }^{63}$

- Catogory 2B: The recommendation is based on lower level evidence, and there is nonuniform consensus that the recommendation should be made. In these instances, because the evidence is not conclusive, institutions take different approaches to the management of a particular clinical scenario. For 
example, based on phase II studies, some institutions may choose to use neoadjuvant therapy in pancreatic cancer, whereas others may choose to wait for further data. This nonuniform consensus does not represent a major disagreement; rather, it recognizes that given imperfect information, institutions may adopt different approaches. A Category 2B designation should signal to the user that more than one approach can be inferred from the existing data.

- Category 3: The recommendation has engendered a major disagreement among the panel members. The level of evidence is not pertinent in this category because experts can disagree about the significance of high level trials. ${ }^{65}$ Several circumstances can cause major disagreements. For example, if substantial data exist about two interventions but they have never been directly compared in a randomized trial, adherents to one set of data may not accept the interpretation of the other side's results. This situation is seen in the controversy regarding total thyroidectomy versus lobectomy in papillary thyroid cancer. Another situation resulting in a Category 3 designation is when experts disagree about how trial data can be generalized. An example of this is the recommendation for internal mammary node radiation in postmastectomy radiation therapy. One side believed that because the randomized studies included this modality, ${ }^{66}$ it must be included in the recommendation. The other side believed, based on the documented additional morbidity and the role of internal mammary radiation therapy in other studies, that this was not necessary. A Category 3 designation alerts users to a major interpretation issue in the data and directs them to the manuscript for an explanation of the controversy.

Hopefully, the Categories of Consensus inform and reassure users about the basis for the recommendations. It is not a perfect system. In the next year, the NCCN Guidelines Steering Committee will discuss ways to alert guidelines users to the existence of contradictory data and will also consider whether grading the level of evidence further would make the system more serviceable.

\section{References}

1. NationalQuality Forum. A National Framework For Health Care Quality Measurement And Reporting: A Consensus Report. Washington, DC: The National Quality Forum; 2002.
2. Hewitt M, Simons P. Ensuring Quality Cancer Care. Washington, DC: Institute of Medicine; 1999.

3. Grimshaw JM. Effect of clinical guidelines on medical practice: A systematic review of rigorous evaluations. Lancet 1993;342:1317-1322.

4. Smith TJ, Hillner BE. Ensuring quality cancer care by the use of clinical practice guidelines and critical pathways. J Clin Oncol 2001;19:2886-2897.

5. Woolf SH, Grol R, Hutchinson A et al. Clinical guidelines: Potential benefits, limitations, and harms of clinical guidelines. BMJ 1999;318:527-530.

6. Feder G, Eccles M, Grol R et al. Using clinical guidelines. BMJ 1999;318:728-730.

7. Field MJ, Lohr KN, eds. Clinical Practice Guidelines: Direction for a New Program. Washington, DC: National Academy Press; 1990.

8. Eddy DM. Practice policies: What are they?JAMA 1990;263: $877-879$.

9. Capron AM. Practice guidelines: How good are medicine's new recipes? J Law Med Ethics 1995;23:47-48.

10. Winn RJ, Brown NH. The NCCN development process. Oncology 1999;13:28-32.

11. Parke EP, Fink A, Brook RH et al. Physician ratings of appropriate medical indications for six medical and surgical procedures. Am J Pub Health 1986;76:766-772.

12. WoolfSH. Practice guidelines: a new reality in medicine. III. Impact on patient care. Arch Intern Med 1993; 153: 2646-2655.

13. Haldorn DC. Use of algorithms in clinical guideline development. In: Clinical Practice Guideline Development: Methodology Perspectives. Rockville,MD: Agency for Health Care Policy and Research, Public Health Service; 1993:93-104.

14. Parmley W. Clinical practiceguidelines: Does the cookbook have enough recipes? JAMA 1994:272:1374-1375.

15. Osaba D. A taxonomy of the uses of health-related qualityof-life instruments in cancer care and the clinical meaningfulness of the results. Med Care 2002;40(supp13):31-38.

16. Buchan H. Clinical guidelines: Acceptance and promotion. Qual Health Care 1993;2:213-214.

17. Czaja R, McFall SL, Warnecke RB et al. Preferences of community physicians for cancer screening guidelines. Ann Intern Med 1994;120:602-608.

18. Ayanian JZ, GuadagnoliE. Variations in breast cancer treatment by patient and provider characteristics. Breast Cancer Res Treat 1996;40:65-74.

19. Winn RJ, Teng NNH. Clinical practice guidelines in the management of gynecologic malignancies. Hematol Oncol Clin North Am 1999;13:63-75.

20. Earle CC, Neumann PJ, Gelber RD et al. Impact of referral patterns on the use of chemotherapy for lung cancer. J Clin Oncol 2002;20:1786-1792.

21. Cleeland CS, Gonin R, Hatfield AK et al. Pain and its treatment in outpatients with metastatic cancer. N Engl J Med 1994;330:592-596. 
22. Schriefer J. The synergy of pathways and algorithms: Two tools work better than one. J Qual Improve 1994;20:485-499.

23. Brook RH. Practice guidelines and practicing medicine: Are they compatible?JAMA 1989;262:3027-3031.

24. Berg AO, Atkins D, Tierney W. Clinical practice guidelines in practice and education. J Gen Intern Med 1997;12 (Suppl 2):S25-S33.

25. Eddy DM. Anatomy of a decision. JAMA 1990;263:441-443.

26. Holmes-Rovner M, Llewellyn-Thomas H, Entwistle V et al. Patient choice modules for summaries if clinical effectiveness: A proposal. BMJ 2001;323:664-667.

27. Kravitz RL, Melnikow J. Engaging patients in medical decision-making: The end is worthwhile, but the means need to be more practical. BMJ 2001;323:584-585.

28. Grol R. Improving quality of medical care: Building bridges among professional pride, payer profit, and patient satisfaction. JAMA 2001:286:2578-2585.

29. Donabedian A. The quality of care: How can it be assessed? JAMA 1988;260:1743-1748.

30. Agency for Health Care Policy and Research. Using Clinical Practice Guidelines to Evaluate Quality of Care. Washington, DC: ACHPR publication no. 95-0045; March 1995.

31. Weeks J. Outcomes assessment in the NCCN: 1998 update. Oncology 1999;13:69-72.

32. McKee M, Clarke A. Guidelines, enthusiasms, uncertainty, and limits to purchasing. BMJ 1995;310:101-104.

33. Morrison J, Carroll L, Twaddle S et al. Pragmatic randomized controlled trial to evaluate guidelines for the management of infertility across the primary-secondary care interface. BMJ 2001;322:1282-1284.

34. Pauly MV. Practice guidelines: Can they save money? Should they? J Law Med Ethics 1995;23:65-74.

35. Carpenter CE, Nash DB, Johnson NE. Evaluating the cost containment of potential clinical guidelines. Qual Rev Bull 1993;19:119-123.

36. Merlani P, Garnerin P, Diby M et al. Linking guideline to regular feedback to increase appropriate requests for clinical tests: Blood gas analysis in intensive care. BMJ 2001; 323:620-624.

37. Granata AV, Hillman AL. Competing practice guidelines: Using cost-effectivenessanalysis to make optimal decisions. Ann Intern Med 1998;128:56-63.

38. Woolf SH. Practice guidelines, a new reality in medicine. II. Methods of developing guidelines. Arch Intern Med 1992;152:946-952.

39. Audet A-M, Greenfield S, Field M. Medical practice guidelines: current activities and future directions. Ann Intern Med 1990;113:709-714.

40. Eddy DE. Clinical decision-making: From theory to practice: Guidelines for policy statements: the explicit approach. JAMA 1990;263:2239-2240.

41. Shekelle PG, Woolf SH, Eccles $M$ et al. Clinical guidelines: Developing guidelines. BMJ 1999;318:593-596.

42. Shaneyfeldt TM, Mayo-Smith MF, Rothwangl J. Are guidelines following guidelines? The methodological quality of clinical practice guidelines in the peer-reviewed adult literature. JAMA 1999;281:1900-1905.

43. Woolf SH, DiGuiseppi CG, Atkins D et al. Developing evidence-based clinical practiceguidelines: Lessons learned by the US Preventive Services Task Force. Ann Rev Pub Health 1996;17:511-538.

44. Cook DJ, Greengold NL, Ellrodt AG et al. The relationship between systematic reviews and practice guidelines. Ann Intern Med 1997;127:210-216.

45. Browman G. Development and aftercare of clinical guidelines: The balance between rigor and pragmatism. JAMA 2001; 286:1509-1511.

46. Djulbegovic B, Hadley T. Evaluating the quality of clinical guidelines: Linking decisions to medical evidence. Oncology 1998;12:310-314.

47. Winn RJ. NCCN Guideline development: Some lessons learned. Cancer Control 2001;8:11-14.

48. Pearson SD, Margolis CZ, Davis S et al. Is consensus reproducible? A study of an algorithmic guidelines development process. Med Care 1995;33:643-660.

49. Winn RJ, Brown NH, Botnick WZ. Reproducibility of guidelines: A comparison of the NCCN and ASCO lung cancer guidelines. Oncology 1999;13:35-39.

50. American Society of Clinical Oncology. Clinical practice guidelines for the treatment of unresectable non-small cell lung cancer. J Clin Oncol 1997;15:2996-3018.

51. NCCN non-small-cell lung cancer practice guidelines. Oncology 1996;14:81-111.

52. Spratt JS. The risky shift. J Surg Oncol 1991;48:1-3.

53. Fink A, Kosecoff J, Chassin M et al. Consensus methods: Characteristics and guidelines for use. Am J Pub Health 1984;74:979-983.

54. Edmonds JP, Day RO, Bertoch JV. The road to consensus: Considerations for the safe use and prescribing of COX-2-specific inhibitors. Med J Aust 2002;176;332-334.

55. Coulter I, Adams A, Shekelle P. Impact of varying panel membership on ratings of appropriatenessin consensus panels: A comparison of a multi- and single disciplinary panel. Health Serv Res 1995;30:579-589.

56. Grilli R, Magrini N, Penna A et al. Practice guidelines developed by specialty societies: The need for a critical appraisal. Lancet 2000;355:103-106.

57. Glaser EM. Using behavioral science strategies for defining the state-of-the art. J Am Behav Sci 1980;16:79-92.

58. Shekelle P, Eccles MP, Grimshaw JM et al. When should guidelines be updated? BMJ 2001;323:155-157.

59. Shekelle PG, Ortiz E, Rhodes S et al. Validity of the Agency for Healthcare Research and Quality clinical practice guidelines: How quickly do guidelines become outdated? JAMA 2001;286:1461-1467.

60. Choudhry NK, Stelfox HT, Detsky AS. Relationship between authors of clinical practice guidelines and the pharmaceutical industry. JAMA 2002;287:612-617. 
The NCCN Clinical Practice Guidelines in Oncology: A Primer for Users

61. Van der Wyden MB. Clinical practice guidelines: Time to move the debate from the how to the who. Med J Austral 2002;176:304-305.

62. Harbour R, Miller J. A new system for grading recommendations in evidence-based guidelines. BMJ 2001;323:334-336.

63. Baillard DJ, Duncan PW. Role of population-basedepidemiologic surveillance in clinical practice guideline development. In: Clinical Practice Guideline Development: Methodology Perspectives. Rockville, MD: Agency for Health Care Policy and Research, Public Health Service; 1993:93-104.
64. Cook DJ, Guyatt GH, Laupacis A et al. Rules of evidence and clinical recommendations on the use of antithrombotic agents. Chest 1992;102(suppl4):S305-S311.

65. McNeill BJ, Shattuck L. Hidden barriers to the improvement in the quality of care. N Engl J Med 2001;345: 1612-1619.

66. Woolf SH, Lawrence RS. Preserving scientific debate and patient choice: Lessons from the Consensus Panel on mammography screening. JAMA 1997;278:2105-2107. 